\title{
Grippeimpfung und Bedrohung durch die Vogelgrippe: Empfehlungen
}

Prof. Claire-Anne Siegrist, Präsidentin Eidgenössische Kommission für Impffragen (EKIF)

Der Nachweis von H5N1-Influenzaviren in Geflügelbeständen in Rumänien und in der Türkei erhöht das Bedrohungsgefühl durch die Vogelgrippe in der Bevölkerung und generiert viele Fragen. Aus Angst vor Ansteckung mit einem Vogelvirus kommt es in der Bevölkerung zu einer deutlich vermehrten Nachfrage nach dem konventionellen Grippeimpfstoff. Diese könnte die Anzahl der in der Schweiz verfügbaren Dosen übersteigen, mit der Konsequenz, dass für Personen mit erhöhtem Komplikationsrisiko zu wenig Impfstoff vorhanden wäre. Dies wiederum könnte $\mathrm{zu}$ einer Zunahme von Grippetodesfällen im kommenden Winter führen. Diese Sorge macht folgende Stellungnahme erforderlich.

\section{Die Grippeimpfung schützt nicht gegen Vogelgrippe}

Der Impfstoff für die Grippesaison 2005-2006 enthält die drei Virusstämme H1N1, H3N2 und B, die im Februar 2005 als wahrscheinlichste Verursacher der kommenden Grippesaison in der Schweiz identifiziert wurden. Dieser Impfstoff schützt gegen diese drei Stämme, nicht aber gegen den H5N1-Stamm, der gegenwärtig Geflügelbestände befällt.

\section{Die Grippeimpfung wird Personen mit beruflichem Kontakt zu Geflügel und Wildvögeln empfohlen}

Die Vogelgrippe ist sehr gefährlich für Vögel, für Menschen aber nur sehr wenig kontagiös. Bisher wurden weltweit erst 117 Personen mit engem Geflügelkontakt infiziert. Es besteht aber die Befürchtung, dass sich das Vogelgrippevirus H5N1 mit einem humanen Grippevirus (z.B. H1N1 oder H3N2) rekombiniert und auf diese Weise ein Virus entstehen könnte, das für Menschen kontagiös und hochpathogen wäre (Pandemievirus). Ein solches Virus könnte in einer Person entstehen, die in der gleichen Woche von einem Vogelgrippevirus und einem «gewöhnlichen» saisonalen Grippevirus befallen wird. Aus diesem Grund sind Personen mit beruflichem Kontakt zu Geflügel und Wildvögeln aufgefordert, sich gegen die saisonale Grippe impfen zu lassen.

\section{Die Grippeimpfung soll auf Risikogruppen beschränkt werden}

Jährlich sterben in der Schweiz 400 bis 1000 Personen an der Grippe und 1000 bis 5000 werden hospitalisiert, obwohl wirksame und sichere Impfstoffe verfügbar sind. Personen mit erhöhtem Komplikationsrisiko sind klar definiert: 1. Personen im Alter von über 65 Jahren; 2. Erwachsene und Kinder mit erhöhtem Komplikationsrisiko wegen schwerer Grundkrankheit (Herz- und Lungenerkrankungen, Asthma bronchiale, kongenitale Herzvitien, zystische Fibrose, Stoffwechselstörungen [besonders Diabetes mellitus], Niereninsuffizienz, Immunsuppression, Hämoglobinopathien); 3. Erwachsene und Kinder, die regelmässiger medizinischer Betreuung bedürfen und im Verlauf des vorangegangenen Jahres hospitalisiert waren; 4. seit 2005 Personen mit beruflichem Kontakt zu Hausgeflügel und Wildvögeln.

Die Grippeimpfung wird auch den folgenden Bevölkerungsgruppen empfohlen (unter 65 Jahren keine Kostenübernahme durch die Krankenkassen): 1. Bewohnerinnen und Bewohner von Alters- und Pflegeheimen und Patientinnen und Patienten in Einrichtungen für chronisch kranke Personen; 2. Personen, die das Grippevirus auf Risikopersonen übertragen können (Medizinalund Pflegepersonal sowie alle Personen mit direktem Kontakt zu Patientinnen und Patienten und/oder Bewohnerinnen und Bewohnern von Spitälern, Kliniken oder Arztpraxen, bei der Hauspflege, in Alters- und Pflegeheimen sowie in Kurhäusern); 3. Personen, die in nahem Kontakt stehen zu Risikopersonen oder mit ihnen im gleichen Haushalt leben (Kinder inbegriffen).

Aufgrund der Befürchtung, dass ein übermässiger und nicht gerechtfertigter Einsatz der 
Grippeimpfstoffe die Verfügbarkeit für die tatsächlichen Risikogruppen gefährden könnte, empfehlen die Eidgenössische Kommission für Impffragen (EKIF) und das BAG übereinstimmend Ärztinnen und Ärzten, vorzugsweise Perso- nen mit erhöhtem Komplikationsrisiko zu impfen. Mit Ausnahme von Personen mit beruflichem Vogelkontakt sind es die gleichen Risikogruppen wie in den vergangenen Jahren.

\section{Menace de grippe aviaire et vaccination contre la grippe: recommandations}

Prof. Claire-Anne Siegrist, présidente de la Commission fédérale pour les vaccinations (CFV)

La découverte récente de virus H5N1 dans les volailles en Roumanie et en Turquie accentue la perception de la menace de grippe aviaire dans la population générale et génère de nombreuses questions. Dans la crainte d'une contamination par un virus aviaire, la demande d'une vaccination contre la grippe se fait de plus en plus fréquente dans la population. Cette demande pourrait dépasser le nombre de doses de vaccin disponibles en Suisse pour la vaccination des personnes à risques élevés de complication, augmentant ainsi considérablement le nombre de décès dus à la grippe saisonnière. Cette préoccupation motive la mise au point suivante.

\section{La vaccination contre la grippe ne protège pas contre la grippe aviaire}

Le vaccin de la saison 2005-2006 contient les trois souches virales H1N1, H3N2 et B considérées en février 2005 comme étant les plus susceptibles de circuler dans notre pays pendant la saison d'hiver 2005-2006. Ces vaccins sont efficaces contre ces trois souches mais les défenses immunitaires qu'ils déclenchent ne protègent pas contre la souche H5N1 qui affecte actuellement les volailles.

Correspondance:

Secrétariat CFV

Office fédéral de la santé publique

Section Vaccinations

Case postale

CH-3003 Berne

Tél. 0313238706

Fax 0313238795

E-mail: epi@bag.admin.ch

\section{La vaccination contre la grippe est recommandée aux personnes en contact professionnel avec la volaille et les oiseaux sauvages}

La grippe aviaire est très dangereuse pour les oiseaux mais très peu contagieuse pour l'homme, seules 117 personnes en contact étroit avec des volailles ayant été infectées. La crainte est que le virus $\mathrm{H} 5 \mathrm{~N} 1$ de la grippe aviaire puisse se combiner avec un virus de la grippe humaine (comme H1N1 ou H3N2) et donner alors naissance à un nouveau virus très contagieux et dangereux pour l'humain (virus pandémique). Un tel virus pourrait apparaître chez une personne infectée la même semaine par les virus de la grippe aviaire et par celui de la grippe saisonnière. C'est pourquoi les professionnels en contact avec de la volaille et des oiseaux sauvages sont invités à se faire vacciner contre la grippe saisonnière.

\section{La vaccination contre la grippe doit être réservée aux personnes des groupes à risques}

Chaque année, 400 à 1000 personnes meurent de la grippe en Suisse et 1000 à 5000 sont hospitalisées, alors que des vaccins efficaces et sûrs existent contre cette infection. Les personnes à risques élevés de complications de la grippe sont bien définies: 1. les personnes âgées de plus de 65 ans; 2 . les adultes et enfants exposés à des risques accrus de complications en raison de maladies graves (maladies cardiaques ou pulmonaires chroniques, asthme bronchique, malformations cardiaques congénitales, mucoviscidose, troubles métaboliques chroniques [notamment le diabète], insuffisance rénale, hémoglobinopathie ou immunosuppression); 3. les adultes et enfants qui requièrent un suivi médical régulier et qui ont été hospitalisés au cours de l'année précédente, 4 . depuis 2005 , les personnes en contact professionnel avec de la volaille et des oiseaux sauvages. 
La vaccination contre la grippe est également recommandée aux catégories suivantes de la population (sans être remboursée aux personnes de moins de 65 ans): 1 . les résidents de homes et maisons de retraite, les pensionnaires d'institutions pour malades chroniques; 2. les personnes qui peuvent transmettre le virus de la grippe à des personnes à risques (personnel médical et soignant ainsi que toute personne en contact direct avec les patients et/ou résidents des hôpitaux et cliniques, des cabinets médicaux, des services de soins à domicile, des homes pour personnes âgées, des établissements de soins ou de cure); 3 . les personnes qui sont en contact proche ou vivent en ménage commun avec des personnes à risques (enfants y compris).

Craignant qu'une utilisation aberrante des vaccins contre la grippe disponibles en Suisse pour la saison 2005-2006 ne prive les patients à risques élevés de la protection nécessaire, la Commission fédérale pour les vaccinations en accord avec l'Office fédéral de la santé publique recommandent aux médecins de vacciner prioritairement les personnes à risques élevés de complications. A l'exception des personnes en contact professionnel avec de la volaille et des oiseaux sauvages, ces groupes à risques sont actuellement les mêmes qu'au cours des années précédentes. 